\title{
ANÁLISE DA EFICIÊNCIA TÉCNICA DE PROPRIEDADES CITRÍCOLAS DO ESTADO DE SÃO PAULO
}

\author{
Felippe Clemente* \\ Marília Fernandes Maciel Gomes ${ }^{\dagger}$ \\ Viviani SiLva Lírio ‡
}

\begin{abstract}
Resumo
Este trabalho teve por objetivo analisar a eficiência técnica das propriedades citrícolas do estado de São Paulo. Aplicou-se a análise envoltória de dados (DEA), para calcular os níveis de eficiência técnica, e uma abordagem econométrica, para conhecer os determinantes da eficiência técnica. Os resultados mostraram que grande parte das propriedades citrícolas atua de forma ineficiente e as variáveis que mais contribuem para aumento da eficiência são "escolaridade" e "tempo como produtor rural". As funções de produção média e de fronteira mostraram-se diferentes quanto à magnitude dos seus parâmetros, indicando que esse instrumental de análise é muito importante e sua estimação e interpretação, de forma correta, pode facilitar a tomada de decisão e melhorar significativamente a alocação dos recursos.
\end{abstract}

Palavras-chave: Eficiência técnica; Setor citrícola; DEA.

\begin{abstract}
This study aimed at analyzing the technical efficiency of citrus farms in the State of São Paulo.For that, data envelopment analysis was applied (DEA) to calculate the levels of technical efficiency, and an econometric approach was used to know the determinants of technical efficiency. The results showed that the majority of citrus properties of São Paulo operate inefficiently and the variables that contribute most to increasing efficiency are "schooling" and "time as farmers." The average production and frontier functions were different regarding the magnitude of its parameters, indicating that the instrumental analysis is very important and its correct estimation and interpretation, can facilitate decision making and significantly improve the allocation of resources.
\end{abstract}

Keywords: Technical efficiency; The citrus sector; DEA.

JEL classification: Q12, C25

DOI: http://dx.doi.org/10.1590/1413-8050/ea 104786

\footnotetext{
* Universidade Federal de Viçosa - MG. E-mail: felippe.clemente@ufv.br

† Universidade Federal de Viçosa — MG. E-mail: mfmgomes@ufv.br

‡ Universidade Federal de Viçosa — MG. E-mail: vslirio@ufv.br
} 


\section{Introdução}

A cultura da laranja está presente em todos os estados brasileiros em padrões de produção bastante distintos. Ao mesmo tempo, a produção de laranjas é uma atividade concentrada espacialmente no país, uma vez que $96 \%$ da produção provêm de apenas seis estados, com destaque para São Paulo, responsável por 78\% da produção Agrianual (2010).

De acordo com Neves (2005), no contexto do agronegócio brasileiro, o setor citrícola se destaca, pois envolve mais de 24 mil propriedades rurais e emprega diretamente $11,2 \%$ da força de trabalho agrícola do estado de São Paulo e 2,2\% do Brasil. No ano de 2010, o Brasil foi o principal produtor de laranja do mundo, com $31 \%$ da produção, seguido pelos Estados Unidos e pela União Européia, com $16 \%$ e $11 \%$, respectivamente. Nas vendas externas o posto ocupado é equivalente: o país é o principal exportador de suco de laranja concentrado congelado (SLCC), com cerca de $80 \%$ do mercado internacional. Adicione-se a esta informação o fato de que as exportações de suco de laranja se mantêm, desde 1994, entre 1,1 e 1,4 milhão de toneladas, gerando mais de um bilhão de dólares de divisas. (Neves 2005).

Com relação à produção de laranja no estado de São Paulo, apesar da importância relativa, observa-se que a produção não se distribui uniformemente entre os citricultores, havendo uma discrepância entre o número de produtores e a quantidade produzida. De acordo com Clemente (2010), a estrutura de produção de laranja no interior paulista caracteriza-se "por muitos produzem pouco" e "poucos produzem muito". Os produtores de até 100 hectares correspondem a $48 \%$ do número total de produtores, mas respondem por apenas $17,5 \%$ da produção. No outro extremo, os produtores com mais de 300 hectares correspondem a apenas $17 \%$ do número total de produtores, porém respondem com $43,3 \%$ da produção paulista. A partir dessas informações acredita-se haver espaço para investigar a existência de uma possível ineficiência na produção de laranjas em São Paulo.

Na verdade, o conceito de eficiência é relativo e difere dos conceitos de eficácia e produtividade. A eficácia está ligada apenas ao que é produzido, sem levar em conta os recursos usados para a produção. A produtividade é denominada pela razão entre o que foi produzido e o que foi gasto para produzir. Já a eficiência compara o que foi produzido, dados os recursos disponíveis, com o que poderia ter sido produzido com os mesmos recursos, de modo que, caso a unidade produtiva esteja muito distante desse parâmetro, ela pode ser considerada ineficiente. Existem duas formas de uma unidade não eficiente tornar-se eficiente: a primeira é reduzindo os insumos, mantendo constante a produção; a segunda é aumentando a produção, mantendo constantes os insumos (Mello et al. 2005).

Em termos aplicados, a análise de eficiência de unidades produtivas tem importância tanto para fins estratégicos (comparação entre unidades produtivas), quanto para o planejamento (avaliação dos resultados do uso de diferentes combinações de fatores) e para a tomada de decisão (como melhorar o desempenho atual, por meio da análise da distância entre a produção atual e potencial).

Nesse contexto, o trabalho tem por objetivo analisar a eficiência técnica de propriedades citrícolas do estado de São Paulo, tendo como base de informações o período compreendido entre os anos de 2009 e 2010, fase em que foram realizadas as entrevistas com citricultores para obter informações a respeito 
da produção de laranjas em cada propriedade.

Para alcançar o objetivo, o artigo está estruturado em quatro seções, além desta introdução. Na segunda seção, apresenta-se o referencial teórico do trabalho e na terceira seção o referencial analítico. Na quarta e na última seção mostra os principais resultados obtidos e as conclusões do estudo, respectivamente.

\section{Referencial Teórico}

Segundo Carvalho (1984), normalmente, em análises microeconômicas, representa-se a função de produção como sendo uma relação entre $y$, a quantidade produzida do bem e um conjunto de parâmetros, $X_{1}, X_{2}, \ldots, X_{n}$, que identificam as quantidades utilizadas de diversos fatores, respeitando o processo de produção mais eficiente. Em outras palavras, trata-se de "uma relação técnica, que associa a cada dotação de fatores de produção a máxima quantidade de produto obtida a partir da utilização desses fatores" (Barbosa 1985).

Nesse âmbito, a questão da eficiência ganha contornos importantes. Para Sato (1975), a agregação de funções de produção e a sua subsequente estimação econométrica com vistas à geração de uma macro função de produção, sem levar em conta as diferenças na eficiência produtiva, geram resultados viesados. Tais, resultados, ao serem utilizados pelos agentes responsáveis pelo processo produtivo, podem comprometer a alocação eficiente dos recursos, os quais, na maioria das vezes, são escassos e caros.

Em outras palavras, comparando diferentes unidades de produção, podese incorrer em erro, caso a análise se baseie apenas na estimação da função de produção média. Isso acontece porque existem diferenças na utilização dos fatores de produção, os quais geram níveis diferentes de eficiência técnica da produção. Assim, para estimar corretamente a função de produção agregada para uma determinada região ou estado, é preciso eliminar as ineficiências existentes em cada unidade produtiva, ou considerá-las adequadamente nas análises pretendidas. Nesse sentido, torna-se necessário estimar uma função de produção de fronteira que caracteriza a melhor tecnologia (best pratice), a partir da qual pode-se fazer comparações entre unidades de produção em termos de eficiência produtiva e estrutura da tecnologia de produção.

A Figura 1 ilustra a diferença entre uma função de produção média estimada por mínimos quadrados e uma função de produção de fronteira. Percebese que na função média, ao minimizar o quadrado dos desvios, existem pontos acima e abaixo da função. Já na função de fronteira, todos os pontos situam-se nela, ou abaixo. Os pontos que se encontram em cima da fronteira referem-se às unidades eficientes. De modo equivalente, os pontos abaixo da fronteira apresentam algum tipo de ineficiência (Fare et al. 1994).

Assim, a existência de ineficiência impossibilita a estimação correta da função, uma vez que contradiz todos os princípios microeconômicos de maximização de lucro. Nesse sentido, é necessário eliminar as ineficiências, no intuito de projetar as unidades ineficientes para a fronteira eficiente. Feito isso, podese estimar a função de produção, a qual expressará melhor as relações entre insumos e produto, já desprovida de ineficiência. 


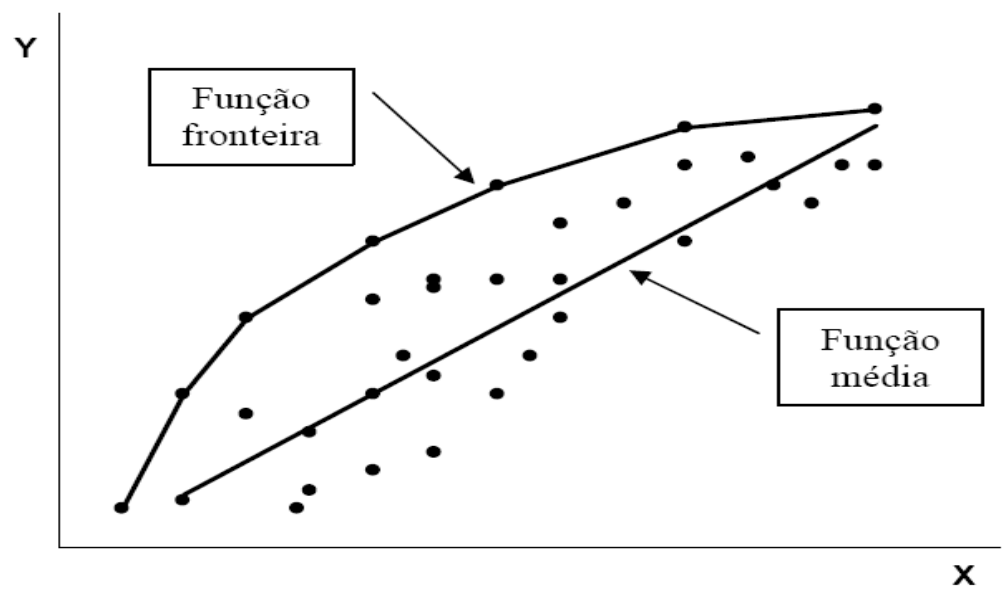

Fonte: Gomes (2009)

Figura 1: Representação da função de produção

\section{Metodologia}

\subsection{Análise envoltória de dados}

Em uma estrutura produtiva, as quantidades máximas de produtos que podem ser obtidas, dados os insumos utilizados, determinam a fronteira de produção (Lins \& Meza 2000). A produção de laranja, assim como outras atividades agrícolas, envolve sistemas de produção muito variáveis o que torna mais complexa a tomada de decisão acerca da melhor alocação dos recursos.

A análise envoltória de dados (DEA) é uma técnica não paramétrica que se baseia na programação matemática para analisar a eficiência relativa de unidades produtoras. Na literatura relacionada com modelos DEA, uma unidade produtora é tratada como uma unidade tomadora de decisão (DMU, decision making unit), uma vez que desses modelos provém uma medida para avaliar a eficiência relativa de DMUs (Gomes 2009).

Na DEA, o termo análise envoltória deriva do fato de, nessa abordagem, a análise ter como referência as DMUs outliers, buscando detectar as DMUs eficientes e construir um plano geométrico de apreciação dessas unidades.

De acordo com Ferreira (2005), a diferença fundamental entre a abordagem DEA e a análise paramétrica, como a fronteira estocástica, está no fato de a primeira ser não paramétrica, estimando uma fronteira determinística, e a segunda ser paramétrica, com base em função estocástica.

Uma significativa limitação ao uso da abordagem paramétrica para mensuração da eficiência decorre do fato de que ela requer que se conheçam, ou se utilizem meios para estimar, estatisticamente, a função de produção considerada. Devido à complexidade das relações de mercado, a estimação da forma funcional apropriada para diferentes DMUs nem sempre é possível. Assim, o enfoque não paramétrico, que utiliza a programação matemática, a exemplo de DEA, parece ser mais apropriado.

Outra considerável vantagem da DEA em relação à estimação paramétrica está na identificação individualizada de cada produtor no quesito eficiência, o que é possível através dos escores de eficiência gerados pela operacionalização do modelo. 
Essas características conferem ao método uma potencialidade de explicar, com maior propriedade e com pouca interferência dos avaliadores, as complexidades inerentes às condições reais (Ferreira 2005).

Apesar das vantagens apresentadas, essa metodologia também apresenta desvantagens, dentre as quais se destaca a sensibilidade à presença de outliers e à inclusão ou exclusão de uma ou mais unidades no conjunto de observações, o número de variáveis consideradas na análise, a impossibilidade de se testar estatisticamente os resultados e ainda, a desconsideração da presença de fatores aleatórios e erros de medição, de forma que toda a distância para a fronteira é considerada devido à ineficiência (Nascimento 2013).

Metodologias alternativas ao DEA foram desenvolvidas com objetivo de avançar nos estudos sobre eficiência. Nesse sentido, Aigner et al. (1977) propuseram uma função de produção específica para dados "cross-section", em que os desvios observados em relação à função de produção poderiam ser devidos à ineficiência produtiva e aos efeitos aleatórios. Essa função é denominada fronteira de produção estocástica (SFA).

O modelo SFA possui vantagens, sendo que as principais são a pouca sensibilidade a problemas de erros de medida, a estimação de intervalos de confiança para os coeficientes de eficiência e a desobrigatoriedade de assumir uma hipótese a respeito de retornos de escala, e desvantagens, como o fato de poder sofrer dos mesmos problemas tradicionais de análise de regressão, de limitações relacionadas à omissão de variáveis, a possível autocorrelação dos erros, a heterocedasticidade e endogeneidade (Nascimento 2013).

Dessa forma, constata-se que não existe consenso na literatura especializada justificando a escolha da DEA ou de fronteiras estocásticas, já que ambas têm vantagens e desvantagens. A escolha do modelo DEA, para este trabalho, deveu-se, principalmente, ao tamanho da base de dados da análise.

No modelo DEA, segundo Charnes et al. (1994), para estimar e analisar a eficiência relativa das DMUs, utiliza-se a definição de ótimo de Pareto, segundo o qual nenhum produto pode ter sua produção aumentada sem que sejam aumentados os seus insumos ou diminuída a produção de outro produto. A eficiência é analisada, relativamente, entre as unidades.

Para incorporar a natureza multiproduto e multi-insumo da produção, Charnes et al. (1994) propõe a técnica DEA para a análise das diferentes unidades, quanto à eficiência relativa.

A função distância ${ }^{1}$ é empregada para incorporar a natureza multiproduto e multi-insumo na análise de produtividade e eficiência, sem a necessidade de especificar objetivos comportamentais dos tomadores de decisão.

De acordo com Fare et al. (1994), a forma conveniente de descrever a característica multiproduto da produção é pela tecnologia de produção, definida pelo conjunto S, representado na equação 1:

$$
S=\{(x, y): x \text { pode produzir } y\}
$$

que é definido pelo conjunto de todos os vetores de insumos e produtos $(x, y)$, tal que $x$ possa produzir $y$, em que $x$ é um vetor $(k \times 1)$ não negativo de insumos e $y$, um vetor ( $m \times 1)$ não negativo de produtos.

\footnotetext{
${ }^{1}$ Pode ser definida como orientação insumo ou orientação produto. A orientação insumo caracteriza a tecnologia de produção pela minimização proporcional (contração) do vetor insumo, dado um vetor de produto. Já a orientação produto caracteriza a tecnologia de produção pela maximização proporcional do vetor produto, dado um vetor de insumo.
} 
O conjunto de tecnologias de produção pode, de forma equivalente, ser definido pelo conjunto de possibilidades de produção $P(x)$, que representa o conjunto de todos os vetores de produtos $y$, que pode ser produzido pelo vetor de insumos $x$, isto é,

$$
P x=\{y: x \text { pode produzir } y\}
$$

A função distância com orientação produto, de acordo com Shephard (1970), pode ser definida pelo conjunto de produtos $P(x)$, como

$$
\begin{aligned}
& d_{0}(x, y)=\min \left\{\phi: \frac{y}{\phi} \in P(x)\right\} \\
& d_{0}(x, y)=(\max \{\phi:(\phi y) \in P(x)\})^{-1}
\end{aligned}
$$

em que $\phi$, na expressão 3, é um fator mínimo, pelo qual o produto pode ser contraído e, ainda assim, pertencer ao conjunto de possibilidades de produção.

A função distância $d_{0}(x, y)$ poderá ter valores menores ou iguais a 1 , se o vetor de produto $y$ for um elemento do conjunto de possibilidade de produção $P(x)$; se for igual a $1,(x, y)$ estará sobre a fronteira tecnológica; nesse sentido, a produção será tecnicamente eficiente.

O modelo DEA com orientação-produto e pressuposição de retornos não constantes à escala procura maximizar o aumento proporcional nos níveis de produto, mantendo fixa a quantidade de insumos. De acordo com Charnes et al. (1994), pode ser representado algebricamente por:

$$
\begin{array}{r}
{\left[d_{0}(x, y)\right]^{-1}=M A X_{\theta, \lambda, S^{+}, S^{-}} \phi} \\
\text { sujeito a } \\
\phi y_{i}-Y \lambda+S^{+}=0 \\
-x_{i}+X \lambda+S^{-}=0 \\
N 1^{\prime} \lambda \leqslant 1 \\
\lambda \geqslant 0 \\
S^{+} \geqslant 0 \\
S^{-} \geqslant 0
\end{array}
$$

em que $Y_{i}$ é um vetor $(m \times 1)$ de quantidades de produto da $i$-ésima DMU; $x_{i}$ é um vetor $(k \times 1)$ de quantidades de insumo da $i$-ésima DMU; $Y$ é uma matriz $(n \times m)$ de produtos das $n$ DMUs; $X$ é uma matriz $(n \times k)$ de insumos das $n$ DMUs; $\lambda$ é um vetor $(n \times 1)$ de pesos; $N 1$ é um vetor $(n \times 1)$ de números uns; $S^{+}$é um vetor de folgas relativo aos produtos; $S^{-}$é um vetor de folgas relativos aos insumos; e $\phi$ é um escalar que tem vetores iguais ou maiores do que 1 e indica o escore de eficiência das DMUs, ou seja, um valor igual a 1 indica eficiência técnica da $i$-ésima DMU, em relação às demais, enquanto um valor maior que 1 evidencia a presença de ineficiência técnica relativa. $\mathrm{O}$ problema apresentado em 4 é resolvido $n$ vezes - uma vez para cada DMU, e, como resultado, apresenta os valores de $\phi$ e $\lambda$, sendo $\phi$ o escore de eficiência da DMU sob análise e $\lambda$ fornece os peers (as DMUs eficientes que servem de referência para a $i$-ésima DMU ineficiente). 


\subsection{Modeto Tobit}

Para verificar quais variáveis estão associadas à eficiência e à ineficiência das propriedades citrícolas do estado de São Paulo, utiliza-se o modelo econométrico Tobit.

Segundo Ferreira (2005), o modelo Tobit é utilizado nos casos em que a variável dependente está compreendida entre certos valores ou concentrada em pontos iguais a um valor-limite. Conforme Vasconcellos \& Alves (2000), isso se dá em decorrência de os fatores ligados à mensuração dos dados não possibilitarem a representação de valores da variável de respostas para toda a sua extensão. Nessas situações, a aplicação do modelo Tobit objetiva contornar o problema da censura valendo-se de técnicas estatísticas que possibilitem fazer inferências para toda a população sem perda de qualidade.

De acordo com Greene (1993), quando se pretende incorporar o erro novamente na equação para se computar a parte estocástica, haverá, em alguns casos, a necessidade de se trabalhar com modelos de variáveis censuradas ou, como mais conhecido, modelo Tobit cujo nome se deve a James Tobin, seu criador.

O modelo Tobit é estimado da seguinte forma:

$$
Y_{i}^{*}=\beta X_{i}^{\prime}+\mu_{i}
$$

onde $\mu_{i}$ é iid $\approx \mathrm{N}\left(0, \sigma^{2}\right)$.

Os valores observados de $Y_{i}$ são chamados de $Y^{*}$, ou seja, são condicionadas a:

$$
\begin{aligned}
& Y_{i}=Y^{*} s e Y_{i}^{*} \geqslant Y_{0} \\
& Y_{i}=Y_{0} s e Y_{i}^{*} \leqslant Y_{0}
\end{aligned}
$$

Conforme colocado por Amemiya (1984), a função de ML para o modelo Tobit é:

$$
L=\prod_{0}\left[1-\phi\left(X^{\prime} \alpha\right)\right] \prod_{1} \sigma^{-1} \phi\left[\left(\frac{Y_{i}}{\sigma}-X_{i}^{\prime} \alpha\right)\right]
$$

Na equação 7, o primeiro membro representa a probabilidade de o evento ser menor que zero e o segundo termo representa a densidade do evento ser observado. Nos dois termos $\alpha=\frac{\beta}{\sigma}$, pois é uma forma conveniente de escalar a equação para convergência.

Nesse ponto, procura-se concentrar em informações referentes às características das propriedades (tamanho e número de funcionários) e característica do produtor (idade, escolaridade e tempo como produtor). Espera-se que essas variáveis impactem positivamente no fato das propriedades serem eficientes. Assim a seguinte equação foi estimada, com base nos dados primários obtidos com uma amostra de citricultores do estado de São Paulo:

$$
Y_{i}=\beta_{1}+\beta_{2} I_{i}+\beta_{3} E_{i}+\beta_{4} T e_{i}+\beta_{5} T_{i}+\beta_{6} N f_{i}+\epsilon_{i}
$$

em que:

$Y_{i}=$ escores de eficiências obtidos por meio da Análise envoltória de dados. Logo, cada DMU tem um coeficiente positivo de eficiência, limitada ao intervalo de 0 a 1 ; 
$I_{i}=$ idade do produtor (em anos);

$E_{i}=$ escolaridade do produtor (em anos);

$T e_{i}=$ tempo como produtor rural (em anos);

$T_{i}=$ tamanho da propriedade (em hectares);

$N f_{i}=$ número de funcionário nas propriedades;

$\epsilon_{i}=$ termo de erro.

A estimação da equação 8 permite fazer inferências para toda a população sem perda de qualidade.

Espera-se que todas essas variáveis impactem positivamente na eficiência da propriedade. As variáveis idade e tempo como produtor rural evidenciam a experiência do produtor no ramo da citricultura. A variável "escolaridade" indica o nível de conhecimento técnico do citricultor, e a variável "tamanho da propriedade" transfigura na produção de laranjas concretizada.

\subsection{Função de produção}

Para analisar a produção, utiliza-se a função de produção tipo Cobb-Douglas, definida da seguinte forma:

$$
Y=A X_{1}^{\beta_{1}} X_{2}^{\beta_{2}} X_{3}^{\beta_{3}}
$$

em que $Y$ é a variável dependente relacionada à produção; $A$ indica o nível tecnológico; $X_{i}$ refere-se às variáveis independentes, representando os fatores de produção terra, trabalho e capital; e os $\beta_{i}$ são os parâmetros a serem estimados.

Aplicando-se logaritmo em ambos os lados da equação, define-se a forma funcional da função de produção média, descrita pelo seguinte modelo de regressão:

$$
\log Y_{i}=\log A+\beta_{1} \log X_{1 i}+\beta_{2} \log X_{2 i}+\beta_{3} \log X_{3 i}+\mu_{i}
$$

em que os $\beta$ s referem-se aos parâmetros a serem estimados; e $\mu_{i}$ representa o termo de erro aleatório, que se pressupõe ter média 0 e variância constante. Ao expressar a função dessa forma, os coeficientes $\beta_{i}$ passam a ser as elasticidades de produção de cada fator.

Nessa função de produção, estimada com os dados originais, existem ineficiências relativas entre os produtores da amostra. Com isso, após calcular o escore de eficiência para cada produtor, os valores projetados das variáveis são utilizados para estimar uma nova função de produção agregada, já desconsideradas as ineficiências na alocação de recursos no processo produtivo. Essa nova função, denominada de função de produção de fronteira, foi estimada de forma semelhante à função média, diferindo apenas nas variáveis utilizadas, isto é,

em que:

$$
\log Y_{i}^{P}=\log A+\beta_{1} X_{1 i}^{P}+\beta_{2} X_{2 i}^{P}+\beta_{3} X_{3 i}^{P}+\mu_{i}
$$

$$
\begin{aligned}
Y_{i}^{P} & =\phi Y_{i}+S_{i}^{+} \\
X_{1 i}^{P} & =X_{1 i}-S_{1 i}^{-} \\
X_{2 i}^{P} & =X_{2 i}-S_{2 i}^{-} \\
X_{3 i}^{P} & =X_{3 i}-S_{3 i}^{-}
\end{aligned}
$$


em que $\phi_{i}$ é o escore e eficiência estimado por meio do modelo de envoltória apresentado em 4 para o $i$-ésimo produtor e $S_{j} i$ refere-se à folga na $j$-ésima variável estimada para cada produtor.

\section{4 Área de Estudo}

Com a finalidade de investigar o nível de produção de laranja dos citricultores bem como a quantidade de insumos utilizados, foram realizadas entrevistas com produtores de laranja do estado de São Paulo previamente selecionados para aplicação de um questionário estruturado, no período de dezembro de 2009 a fevereiro de 2010. Foram entrevistados citricultores de onze cidades do interior do estado de São Paulo, região que concentra a maior produção de laranja do país.

O estado de São Paulo possuía, em 2006, aproximadamente 6300 citricultores ativos, concentrando $81 \%$ de toda a produção nacional de laranja.

Neves (2005) evidencia que as regiões citrícolas em São Paulo dividem-se em norte e nordeste, centro, sul e sul novo (Figura 2). A primeira compreende a região de Bebedouro e Barretos, São José do Rio Preto e Votuporanga e a região de Catanduva, responsável por $45 \%$ da produção do estado. Na segunda, participam a região de Araraquara e Matão, Itápolis e Taquaritinga, representando $30 \%$ da produção. No sul, fazem parte a região de Limeira, Avaré/Botucatu e Itapetininga. Já o sul novo contempla de Bauru a Itapetininga. Essas duas últimas regiões concentram $25 \%$ da produção (Tavares 2006).

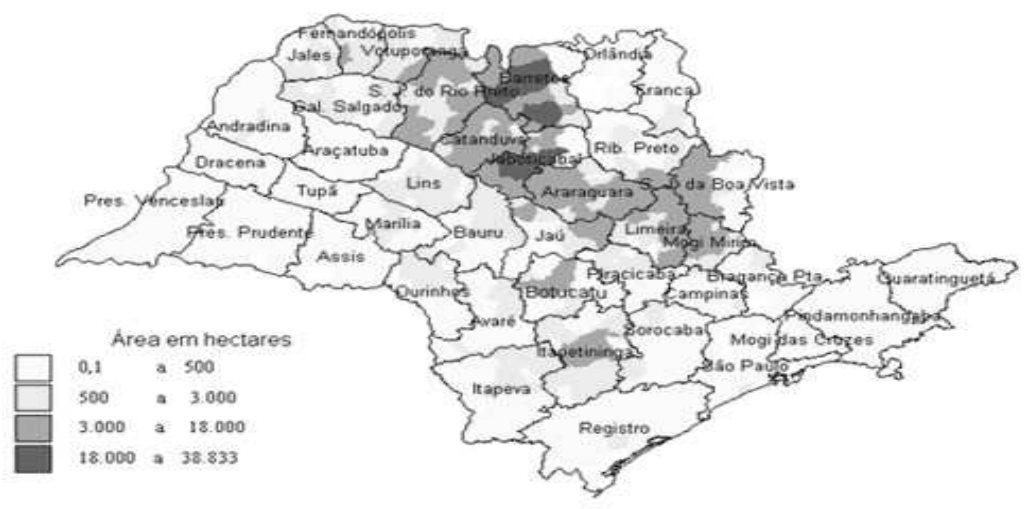

Fonte: Alicitros (2009)

Figura 2: Principais regiões produtoras do estado de São Paulo

Ao se estruturar a amostra, o objetivo foi selecionar citricultores das regiões onde mais se concentra a produção de laranja do estado de São Paulo. O cálculo do tamanho da amostra ${ }^{2}$, com um intervalo de confiança e tolerância do erro amostral de 10\%, resultou em 67 questionários.

Para a estratificação da amostra, foram utilizadas a participação das regiões na produção de laranja do estado de São Paulo e, em seguida, a produção das principais regiões em 2003. Assim, selecionou-se 30 questionários para a

\footnotetext{
${ }^{2}$ Cálculo do tamanho da amostra: $n=\frac{Z^{2} \cdot p \cdot q \cdot N}{\left[d^{2} \cdot(N-1)+Z^{2} \cdot p \cdot q\right]}($ Greene 1993).
} 
região norte e nordeste, 20 questionários para a região central e 17 questionários para a região sul/sul novo. Como as regiões de Barretos, Catanduva e São José do Rio Preto se localizam no norte e nordeste, foram aplicados 17 questionários para a região de Barretos, 6 para a região de Catanduva e 7 para a região de São José do Rio Preto. Para o centro, os 20 questionários foram aplicados na região de Araraquara e para o sul/sul novo foram aplicados 10 questionários na região de Limeira, 4 na região de Jaú e 3 na região de Bauru.

\subsection{Fonte de dados}

Parte importante da construção da base de dados utilizada nesta pesquisa baseou-se em uma pesquisa exploratória com 67 produtores de laranja localizados em onze municípios paulistas, entre os meses de janeiro e março de 2010. Nessa ocasião, foram levantadas as informações sobre as variáveis utilizadas, como idade, escolaridade e tamanho da propriedade, dentre outras.

Os dados relativos ao custo médio de capital das propriedades do interior do estado de São Paulo e o preço médio da laranja vendida para indústria foram coletados junto ao Instituo de Economia Agrícola (IEA).

\section{Resultados e Discussão}

\subsection{Características descritivas da amostra utilizada}

Para realização da pesquisa, foram aplicados questionários estruturados a 67 produtores de laranja do estado de São Paulo. Ao se estruturar a amostra, foram selecionados produtores dos principais municípios paulistas. O objetivo foi selecionar citricultores das regiões onde mais se concentra a produção de laranja do estado de São Paulo.

Dentre as principais características observadas, notou-se que, no total, $34 \%$ dos entrevistados têm idade entre 23 e 50 anos e $66 \%$ estão com idade acima de 50 anos, o que indica a predominância de produtores com idade mais avançada, o que corrobora a ideia do relativo envelhecimento dos produtores brasileiros, já identificado em outros trabalhos.

Verificou-se, também, razoável nível de escolaridade, já que 33\% dos entrevistados possuem até 5 anos de estudo e $51 \%$ possuem mais de 10 anos de estudo. Em relação ao "tempo como produtor rural", os resultados mostram que $26 \%$ dos entrevistados têm até 20 anos na atividade, $24 \%$ dos produtores têm entre 20 e 30 anos e $50 \%$ produzem a mais de 30 anos, evidenciando a predominância de citricultores com larga experiência na produção da laranja.

A partir da análise do tamanho da propriedade dos citricultores (Figura 3), destaca-se a presença de "pequenas" e "médias" propriedades (até 100 hectares), com $48 \%$. Para $81 \%$ dos produtores, a principal fonte de renda é a agricultura e $55 \%$ obtêm uma renda bruta anual com a laranja acima de $\mathrm{R} \$$ 100.000 (Figura 4).

Quanto ao perfil da mão de obra, os resultados mostraram que $84 \%$ das propriedades possuem mão de obra contratada e apenas $16 \%$ contam com mão de obra exclusivamente familiar. Embora a utilização de mão de obra familiar seja uma característica de pequenas propriedades, verifica-se, também, a presença de mão de obra permanente e remunerada.

Com relação ao número de empregados permanentes, verifica-se que a média de trabalhadores contratados por propriedade é de, aproximadamente, 

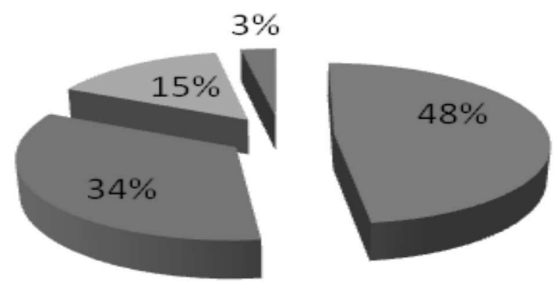

\author{
- De 1 a 100 \\ hectares \\ De 101 a 300 \\ hectares \\ De 301 a 600 \\ hectares \\ - Acima de 600 \\ hectares
}

Fonte: Clemente (2010)

Figura 3: Tamanho da propriedade

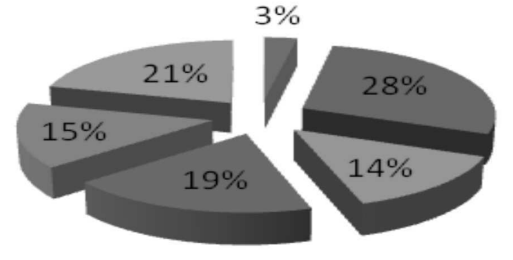

Até R\$ 10.000

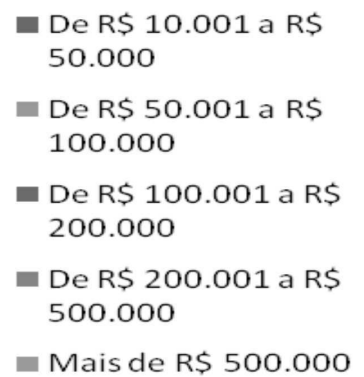

Fonte: Clemente (2010)

Figura 4: Renda bruta anual com a laranja

seis. Porém, a desigualdade da mão de obra entre as fazendas é muito grande, pois há propriedades com até 75 funcionários e propriedades com nenhuma. Isso também é evidenciado pelo alto valor do desvio-padrão $(11,75)$.

Em síntese, os resultados do levantamento primário apontam diferenças significativas nas características produtivas das propriedades citrícolas do estado de São Paulo, com destaque para a escolaridade, a renda bruta e o tempo como produtor rural. Essas divergências podem fomentar algum grau de ineficiência na produção de laranja da região.

\title{
4.2 Análise de Eficiência Técnica
}

De maneira geral, uma análise de eficiência técnica compara o que foi produzido, dados os recursos disponíveis, com o que poderia ter sido produzido com os mesmos recursos. No caso em estudo, as variáveis empregadas no modelo de eficiência para a amostra como um todo, juntamente com suas estatísticas descritivas são apresentadas na Tabela 1. Essas variáveis refletem características da propriedade (produção, tamanho, funcionários e custo de capital) e do produtor (idade, escolaridade e tempo como produtor). Percebese uma relativa diferença de magnitude entre as unidades que compõem a 
amostra, destacando, principalmente, o alto desvio-padrão resultante da relativa dispersão dos dados em torno da média, que enfraquece as inferências de tendência central.

Tabela 1: Estatísticas descritivas das variáveis empregadas no modelo de eficiência dos produtores, 2010

\begin{tabular}{lrrcc}
\hline Variável & \multicolumn{1}{c}{ Média } & Desvio-padrão & Máximo & Mínimo \\
\hline Produção de laranja (cx 40,8kg) & 34920,59 & 39444,87 & 136263,00 & 738,37 \\
Tamanho da propriedade (em ha) & 190,66 & 287,95 & 2100,00 & 12 \\
Número de funcionários & 6,13 & 11,75 & 75 & 0 \\
Custo de capital (em R\$) & 714063,98 & 1049592,85 & 7578522,00 & 46695,12 \\
Idade do produtor (em anos) & 55,21 & 13,11 & 79 & 23 \\
Escolaridade do produtor (em anos) & 9,93 & 5,43 & 17 & 1 \\
Tempo como produtor (em anos) & 31,52 & 14,29 & 63 & 5 \\
\hline
\end{tabular}

Na Tabela 2, é apresentado o sumário estatístico do cálculo da eficiência técnica das unidades que compõe a amostra. Pela média dos escores de eficiência técnica, é possível visualizar o grau de eficiência das propriedades. Já os escores individualizados permitem apontamentos mais específicos a cada unidade produtiva, indicando ineficiência em recursos, bem como apontando DMUs que lhe sirvam de modelo. Essa verificação é importante para analisar em qual situação encontram-se cada propriedade em detrimento do desempenho do conjunto.

Tabela 2: Escores de eficiência técnica das propriedades citrícolas

\begin{tabular}{lccccc}
\hline Variável & Unidades eficientes & Média & Desvio-padrão & Máximo & Mínimo \\
\hline Eficiência técnica & $13,43 \%$ & 0,79 & 0,21 & 1,00 & 0,25 \\
\hline
\end{tabular}

Os resultados demonstram que as propriedades citrícolas de São Paulo apresentam expressivo grau de ineficiência técnica.

A média da eficiência técnica foi de 0,79 , o que sugere a possibilidade de aumento de produção, considerando a mesma proporção de insumos atualmente utilizada, tomando como referência a orientação produto do modelo. Ao analisar as regiões produtoras do estado de São Paulo, evidencia-se que $67 \%$ das propriedades eficientes encontram-se no norte e nordeste do estado. Isso ocorre devido ao fato dessas regiões serem as mais antigas na produção de laranja do país, o que possibilitou aos produtores maior conhecimento quanto à melhor combinação de insumos. Com relação às propriedades mais ineficientes, $71,4 \%$ estão localizadas na região sul e sudeste do estado.

Para comparar o grau de ineficiência da unidade produtiva tomando por base o escore da média de eficiência técnica, foi construído o indicador definido por Ferrier \& Porter (1991), que segue:

$$
\left(\frac{1}{\text { score }}-1\right) \times 100
$$

Assim, é possível dizer que a diferença média de eficiência técnica nas propriedades de laranja requer uma utilização de recursos $26,6 \%$ maior que as propriedades que estão operando sobre a curva de produção. 
Com o objetivo de verificar quais os determinantes da eficiência das propriedades citrícolas do estado de São Paulo, utilizou-se o modelo econométrico Tobit. Os resultados do modelo podem ser observados na Tabela 3.

Tabela 3: Fatores associados à eficiência técnica nas propriedades citrícolas do estado de São Paulo — método Tobit

\begin{tabular}{lccc}
\hline Variável & Coeficiente & Erro-padrão & Valor-p \\
\hline Tamanho da propriedade (em ha) & 0,00021 & 0,00012 & 0,096 \\
Número de funcionários & $-0,0057$ & 0,00292 & 0,054 \\
Idade do produtor (em anos) & 0,0017 & 0,00183 & 0,365 \\
Escolaridade do produtor (em anos) & 0,0283 & 0,00336 & 0 \\
Tempo como produtor rural (em anos) & 0,0039 & 0,00168 & 0,02 \\
Constante & 0,2866 & 0,09289 & 0,003 \\
$\chi_{2}=55,48$ & & \\
\hline
\end{tabular}

Os coeficientes estimados por meio do modelo apresentaram ajustamento satisfatório, conforme verificado pelo nível de significância do teste $\chi^{2}$, que foi significativo em $1 \%$ de probabilidade.

Dentre os fatores que tiveram influência na eficiência técnica, ressalta-se a variável "escolaridade do produtor", que mais impacta na eficiência das propriedades citrícolas. Isso mostra que a capacitação é o principal meio para obter eficiência técnica na produção de laranja.

A variável "tempo como produtor rural" também influencia diretamente a eficiência nas propriedades, indicando que a experiência com a produção é uma variável importante para determinar a eficiência dos citricultores.

A variável "idade", embora importante, não foi significativa. Isso pode ter ocorrido devido à forte relação desta com a variável "tempo como produtor rural". A variável "número de funcionários permanentes" mostrou-se significativa com sinal contrário ao esperado. Já a variável "tamanho da propriedade" foi significativa e positiva, porém com um impacto muito pequeno na determinação da eficiência citrícola.

Com isso, é possível verificar que, para as propriedades produtoras de laranja do estado de São Paulo, além da experiência adquirida como produtor rural, evidencia-se a importância da educação formal que objetiva aprimorar as técnicas dos produtores, promovendo, assim, o aumento da eficiência na produção do fruto.

Embora o uso de modelos Tobit como "segundo estágio", para explicar índices de eficiência provenientes da estimação de fronteiras na DEA, tenha ganhado popularidade nas décadas de 1990 e de 2000, mais recentemente McDonald (2009) demonstrou que seu uso pode ser inapropriado, e que, em tais aplicações, o estimador de Máxima Verossimilhança (ML) é geralmente inconsistente, ao contrário do estimador de Mínimos Quadrados Ordinários (MQO). De forma a constatar ou não essa evidência, estimou-se novamente a regressão, utilizando o método de MQO, conforme Tabela 4.

Observa-se que, embora os valores dos parâmetros sejam bem semelhantes e com os mesmos sinais apresentados na Tabela 3, o erro-padrão e, consequentemente, a significância estatística se mostram bem distintas. Enquanto no primeiro modelo (ML) as variáveis "tamanho da propriedade" e "tempo como produtor rural" foram significativas a $10 \%$, no segundo modelo (MQO) essas passaram a ser não significativas. O contrário ocorre com a variável "idade do produtor", que passa a ser significativa a $15 \%$ no segundo modelo. 
Tabela 4: Fatores associados à eficiência técnica nas propriedades citrícolas do estado de São Paulo - método Mínimos Quadrados Ordinários (MQO)

\begin{tabular}{lccc}
\hline Variável & Coeficiente & Erro-padrão & Valor-p \\
\hline Tamanho da propriedade (em ha) & 0,00028 & 0,00026 & 0,28 \\
Número de funcionários & $-0,0101$ & 0,00617 & 0,106 \\
Idade do produtor (em anos) & 0,0063 & 0,00388 & 0,107 \\
Escolaridade do produtor (em anos) & 0,0145 & 0,0071 & 0,045 \\
Tempo como produtor rural (em anos) & 0,0048 & 0,00354 & 0,178 \\
Constante & 1,391 & 0,19623 & 0 \\
$R^{2}=22,85$ & & & \\
\hline
\end{tabular}

Para verificar possível inconsistência e tendenciosidade do MQO, pensouse em estimar o modelo anterior retirando as observações cujo valor é zero ( $y=$ 0 ) e compará-lo com os parâmetros verdadeiros. Esse resultado encontra-se na Tabela 5. Pode-se observar que as estimativas são diferentes das apresentadas na Tabela 4, o que mais uma vez justifica o uso de um estimador de máxima verossimilhança, que é consistente e usa todas as observações.

Tabela 5: Fatores associados à eficiência técnica nas propriedades citrícolas do estado de São Paulo - método Mínimos Quadrados Ordinários (MQO)

\begin{tabular}{lccc}
\hline Variável & Coeficiente & Erro-padrão & Valor-p \\
\hline Tamanho da propriedade (em ha) & 0,00024 & 0,00027 & 0,373 \\
Número de funcionários & $-0,0085$ & 0,00631 & 0,178 \\
Idade do produtor (em anos) & 0,0059 & 0,00393 & 0,138 \\
Escolaridade do produtor (em anos) & 0,0177 & 0,00744 & 0,021 \\
Tempo como produtor rural (em anos) & 0,0051 & 0,00364 & 0,169 \\
Constante & 1,426 & 0,1993 & 0 \\
$R^{2}=24,66$ & & & \\
\hline
\end{tabular}

\subsection{Função de Produção}

No intuito de eliminar as ineficiências existentes em cada unidade produtiva, foram estimadas funções de produção média e de fronteira do tipo CobbDouglas. A forma funcional utilizada foi escolhida por permitir identificar, diretamente, a elasticidade de produção de um fator, a qual também indica a importância daquele fator no processo produtivo. A função estimada utilizandose os dados originais foi denominada "função média", cujos resultados encontramse na segunda coluna da Tabela 6 .

Conhecendo-se a medida de eficiência e as folgas dos insumos para cada propriedade, elimina-se dos dados originais os excessos de uso dos fatores (ineficiência), obtendo-se novos valores para as variáveis. Com isso, estimouse a "função de produção de fronteira", cujos resultados também se encontram na Tabela 6.

Analisando os resultados das duas funções, nota-se que existem diferenças significativas nos parâmetros da função média e da função fronteira. A variável "terra" mostra-se mais importante na função média, seguida da variável "trabalho". Já para função de produção de fronteira, a variável "trabalho" 
Tabela 6: Coeficientes estimados das elasticidades de produção e $R^{2}$ corrigido para as funções de produção Cobb-Douglas

\begin{tabular}{lccc}
\hline Coeficientes & \multicolumn{2}{c}{ Modelo estimado } & \multirow{2}{*}{ Variação } \\
\cline { 2 - 3 } & Função Média & Função Fronteira & \\
\hline Intercepto & $55,2430^{* * *}$ & $3,0920^{* *}$ & - \\
$\beta_{1}$ (terra) & $6,0120^{* * *}$ & $0,5510^{*}$ & $-91 \%$ \\
$\beta_{2}$ (trabalho) & $0,5740^{*}$ & $0,6530^{*}$ & $14 \%$ \\
$\beta_{3}$ (capital) & $-5,7440^{* * *}$ & $0,1240^{N S}$ & $-102 \%$ \\
$R^{2}$ corrigido & $47 \%$ & $59 \%$ & - \\
\hline
\end{tabular}

evidencia-se mais importante, seguida da variável "terra". Ambas tiveram no capital um fator não significativo.

Embora o trabalho e a terra tenham suas importâncias relativas no processo produtivo, observa-se que existem grandes diferenças na magnitude dos coeficientes entre as duas funções. O coeficiente do fator terra varia em $91 \%$ quando se compara as funções de produção. Já o coeficiente do fator trabalho varia $14 \%$ entre as funções. De certa forma, essas diferenças indicam o tamanho do erro que se pode incorrer na formulação de políticas para o setor caso não utilize uma função de produção corrigida pelas ineficiências. Também, pela análise das diferenças entre os parâmetros das duas funções de produção tem-se uma ideia do impacto da ineficiência na produção.

Gomes \& Baptista (2009) realizaram o cálculo da função média e de fronteira para o setor agropecuário de Minas Gerais. Como principal resultado destaca-se as diferenças significativas nos parâmetros da função média e da função fronteira. De acordo com os autores, observar-se que a função de produção estimada a partir dos dados originais contém parcela significativa de ineficiência produtiva, invalidando a análise. Para saber o real comportamento da produção agrícola é necessário estimar funções de produção de fronteira, que considera os dados desprovidos de ineficiência.

Em relação à elasticidade de escala, percebe-se que o procedimento utilizado para estimar a função de produção possui uma característica importante, que é o fato de impor restrições quanto à homogeneidade da função de produção. Em outras palavras, ao utilizar o modelo de envoltória de dados com pressuposição de retornos não constantes à escala, impõe-se a concavidade da função de produção de fronteira, corrigindo a ineficiência produtiva e impondo restrições na estrutura da tecnologia de produção.

Conforme mostra a literatura, Alves (2002) evidencia que a utilização da função do tipo Cobb-Douglas para produtos agrícolas apresenta uma vantagem do ponto de vista da agregação, devido ao fácil ajustamento. Entretanto, a utilização da função Cobb-Douglas é muito restritiva, pois supõe elasticidade de substituição constante e unitária e só é justificada em casos de multicolinearidade elevada. Para tentar corrigir esse problema, poderia utilizar uma forma mais flexível, a forma translog, que também se torna linear por logaritmos, e é comumente utilizada em aplicações agrícolas (Albuquerque 1987). 


\section{Conclusão}

Neste estudo, objetivou-se analisar a eficiência das propriedades citrícolas do estado de São Paulo, no período de 2009 a 2010. Com isso, aplicou-se a abordagem não paramétrica de análise envoltória de dados para calcular os níveis de eficiência técnica. Para conhecer os determinantes da eficiência técnica dos citricultores, utilizou-se o modelo econométrico Tobit e, posteriormente, para evidenciar as diferenças entre as funções de produção média e de fronteira e auxiliar no processo de tomada de decisão no setor, ajustaram-se funções de produção agregada na forma funcional tipo Cobb-Douglas.

Com base nos resultados, confirma-se a hipótese de que as propriedades citrícolas atuam de forma ineficiente, ou seja, não alocam de forma ótima seus recursos para a produção. Para elevar a eficiência na produção, faz-se necessário aumentar a educação formal dos produtores, de forma a possibilitar maior conhecimento técnico para a produção. As funções de produção média e de fronteira mostraram-se diferentes quanto à magnitude dos seus parâmetros, indicando que esse instrumental de análise é muito importante e sua estimação e interpretação, de forma correta, pode facilitar a tomada de decisão e melhorar significativamente a alocação dos recursos.

Apesar da divergência na literatura acerca do melhor método para o "segundo estágio", constata-se que o uso do método de máxima verossimilhança é o mais adequado para esses dados, haja vista que a variável dependente se apresenta com uma concentração de observações no limite inferior.

Assim, conclui-se que é importante que os agentes envolvidos com o setor produtivo da laranja tenham consciência da existência de ineficiências. Em muitos casos, a redução dessas ineficiências poderia melhorar a alocação dos recursos em todo o setor, ou seja, os insumos utilizados em excesso em algumas propriedades podem ser realocados, aumentando, com isso, o volume total da produção.

Uma sugestão para trabalhos futuros seria o cálculo da função de produção translog e comparação com a função de produção Cobb-Douglas.

\section{Referências Bibliográficas}

Agrianual (2010), Anuário Estatístico da Agricultura Brasileira, Argos, São Paulo.

Aigner, D., Lovell, K. \& Schimidt, P. (1977), 'Formulation and estimation of stochastic frontier production function models', Journal of Econometrics 6, 21-37.

Albuquerque, M. (1987), 'Uma análise translog sobre mudança tecnológica e efeitos de escala: um caso de modernização ineficiente', Pesquisa e Planejamento Econômico 17, 191-220.

Alicitros, A. d. C. d. R. d. L. (2009), 'Histórico da laranja'. URL: http://www.alicitros.com.br/index2/principal.php? $p=$ laranja

Alves, L. (2002), 'Produção agrícola agregada do estado do paraná em 1995', Anhanguera Goiânia 3, 103-121.

Amemiya, T. (1984), 'Tobit models, a survey', Journal of Econometrics 24, 3 61. 
Barbosa, F. (1985), Microeconomia: teoria, modelos econométricos e aplicações à economia brasileira, IPEA/INPES, Rio de Janeiro.

Carvalho, L. (1984), Teoria da firma a produção e a firma: manual de introdução à economia, Saraiva, Sâo Paulo.

Charnes, A., Cooper, W., Lewin, A. \& Seiford, L. (1994), Data envelopment analysis: theory, methodology and application, Kluwer Academic, Dordrecht.

Clemente, F. (2010), 'Viabilidade de implantação de contratos futuros de suco de laranja concentrado congelado no brasil', Viçosa, MG: UFV, IMPRENSA Universitária.

Fare, R., Grosskopf, S. \& Lovell, C. (1994), Productions frontiers, Cambridge University, Cambridge.

Ferreira, M. (2005), 'Eficiência técnica e de escala de cooperativas e sociedade de capital na indústria de laticínios do brasil', Viçosa, MG: UFV, IMPRENSA Universitária.

Ferrier, G. \& Porter, P. (1991), 'The productive efficiency of us milk processing co-operatives', Journal of Agricultural Economics pp. 161-173.

Gomes, A. P. (2009), Introdução à análise envoltória de dados: teoria, modelos e aplicações, Ed. UFV, Viçosa.

Gomes, A. P. \& Baptista, A. (2009), 'Função de produção de fronteira e tomada de decisão na agropecuária', Palestra.

Greene, W. H. (1993), Econometric Analysis, 2 ed. edn, New York: Macmillan. $791 \mathrm{p}$.

Lins, M. \& Meza, L. (2000), Análise envoltória de dados a perspectiva de integração no ambiente de apoio à decisão, COPPE/UFRJ, Rio de Janeiro.

McDonald, J. (2009), 'Using least squares and tobit in second stage dea efficiency analyses', European Journal of Operational Research pp. 792-798.

Mello, J., Meza, L., Gomes, E. \& Neto, L. (2005), 'Curso de análise de envoltória de dados', XXXVII Simpósio Brasileiro de Pesquisa Operacional.

Nascimento, A. (2013), 'Regulação no setor de distribuição de energia elétrica no brasil: análise de metodologias alternativas para a definição dos custos operacionais eficientes', Universidade Federal de Viçosa.

Neves, M. F. (2005), Estratégias para a laranja no Brasil, Atlas S.A., Sâo Paulo.

Sato, K. (1975), Production functions and aggregation, North-Holland publishing company, Amsterdam.

Tavares, M. (2006), 'O mercado futuro de suco de laranja concentrado e congelado: um enfoque analítico', Porto Alegre, RS. UFRGS.

Vasconcellos, M. \& Alves, D. (2000), Manual de econometria: nivel intermediário, Atlas S.A., Sâo Paulo. 